\title{
Adaptive Sliding-Mode Control in Bus Voltage for an Islanded DC Microgrid
}

\author{
Dan Zhang and Jie Wang \\ Department of Electrical Engineering, Shanghai Jiao Tong University, Shanghai 200240, China \\ Correspondence should be addressed to Dan Zhang; nicolezhang1985@126.com
}

Received 4 May 2017; Accepted 27 November 2017; Published 24 December 2017

Academic Editor: Oscar Reinoso

Copyright (c) 2017 Dan Zhang and Jie Wang. This is an open access article distributed under the Creative Commons Attribution License, which permits unrestricted use, distribution, and reproduction in any medium, provided the original work is properly cited.

\begin{abstract}
The control of bus voltage is a crucial task for the stable operation of islanded DC microgrids. To improve the DC bus voltage control dynamics and stability, this paper proposes an adaptive sliding-mode control method based on large-signal model. The slidingmode control, adaptive observation, and fix-frequency pulse width modulation technology are adopted and combined efficiently, which guarantee stable bus voltage and the constant switching frequency of closed-loop system, regardless of how the parameters vary with the variable constant-power loads and uncertainties. In addition, the reference values can be quickly tracked by the state variables using the proposed method without any additional sensors/hardware circuits. Therefore, this method is beneficial for the scalability and plug-play of the distributed generators and loads within the DC microgrids. The performance of the proposed control method has been successfully verified in simulation.
\end{abstract}

\section{Introduction}

The microgrid (MG) has been widely applied in a wide variety of practical fields, such as islands [1,2], remote areas [3], industrial power systems [4], commercial buildings [5], residential homes [6], and university campuses [7]. This kind of technology can yield a high-reliable regional power system with favourable economy and flexible power supply capacity. As a crucial technology of smart grid, the MG can make up the disadvantages existing in centralized power supply grid and alleviate the influence of distributed generators (DGs) fluctuation on utility grid $[8,9]$. Owning to the aforesaid superiorities, the MG has become an excellent candidate for accepting high permeability DGs into the power grid. Compared with the AC MGs, DC MGs can achieve lower expenditure and higher efficiency by eliminating the extra power electronic conversion stages, since DC power is directly utilized by many renewable DGs, energy storage systems, and loads [10]. Additionally, high controllability is offered by DC system, since it does not have issues associated with synchronization between the DGs, reactive power flows, harmonic currents, and DC/AC conversion losses, which are intrinsic to the AC MG [11]. Moreover, the DC MG can run stand-alone and provide reliable power supply to the loads, although it fully decouples from the utility grid. For the aforementioned factors, the DC MG is gaining attention incrementally.

In island mode, the stability of bus voltage is the critical criterion to assess the stability of a DC MG. When an islanded DC MG encounters abrupt changes from loads and DC sources or is required to supply power for constantpower loads (CPLs), Emadi et al. [12,13] shows that the stability of bus voltage will meet greater challenges. Several control strategies have been proposed based on improved PI controllers in $[14,15]$ to improve the stability of bus voltages. In [16], they propose superior linear controllers that perform well in DC distributed networks with CPLs and improve the performance and stability of the system. The aforesaid approaches are linearization control methods with limitations to control accuracy, sensitivity, and robustness to parameter variations. Therefore, the nonlinear control strategies have been increasingly studied in MGs.

In $[17,18]$, the droop and sliding-mode control (SMC) approaches are employed to design controllers, respectively. However, the adopted small-signal models are unable to meet global stability requirements when operating points are 
allowed to work on voltage levels away from the linearization point. In [19], a feedback linearization method is derived on the basis of the Lyapunov stability theory to overcome the impact of CPLs on global stability in DC MG, without considering the uncertainties in the real system. Thus, the robustness of this control system is restricted. Higher order sliding model control (HOSMC) is studied deeply and applied to MGs by Michele [20] and Satish et al. [21]. This method can alleviate chattering and exert the ability of variable structure control for large-signal processing. However, it is difficult to obtain the uncertain boundary and abate the dependence of the control system on the model, which limit the application of this approach in engineering. In order to ensure robustness of control systems under loads and sources variations, a sliding-mode control method based on a washout filter with hysteresis band $(\mathrm{HB})$ is proposed [22, 23]. The disadvantages of this technique are that there exist variable switching frequency and high sensitivity to noise. Su et al. [24] employ an adaptive total sliding-mode control (ATSMC) to ensure the stability and dynamic performances of the MG. The advantages of SMC in design and its robustness for parameter uncertainties and external disturbance are shown obviously. In [25], an adaptive nonlinear observation method is proposed for a boost power factor correction (PFC) converter to estimate the load value without load information. This method offers a reliable and cost-effective solution with minimized sensors. In [26], by employing PWM technique in SMC, a fixed-frequency pulse width modulation- (PWM-) based SM controller can be obtained. The frequency of switching signal is constant, regardless of how the duty cycle varies with control signal. The antecedent control methods used in MGs have been well studied and achieved positive control effects. However, single method is incapable of solving hybrid issues, such as variable structure characteristics existing in converters, frequency fluctuation, system parameter variation, and disturbance caused by CPLs. Therefore, it is necessary to associate with several control methods to iron out complex problems.

The main contributions of this paper are as follows. The first one is that an advanced bus voltage controller based on adaptive sliding-mode control (ASMC) method combined with fixed-frequency PWM technology using large-signal modelling theory is proposed. Second contribution is to apply the proposed control method to an islanded DC MG with CPLs. Under uncertain external disturbances, the adaptive observer can accurately estimates the values of loads and battery voltage without any additional sensors/hardware circuits. Therefore, it is beneficial for the scalability and plugplay of the DGs and loads within the DC MG. The global SMC method can force the state variables to track their reference values quickly and stably in the presence of CPLs by controlling bidirectional buck/boost converter. Also, fix-frequency PWM is adopted to make the switching frequency constant, which greatly simplifies the design complication of the filter compared with HB method. The third contribution is that the performance of the proposed controller has been validated through theoretical proof and simulation studies in MATLAB. The proposed composite control method offers a very reliable and cost-effective solution for various applications

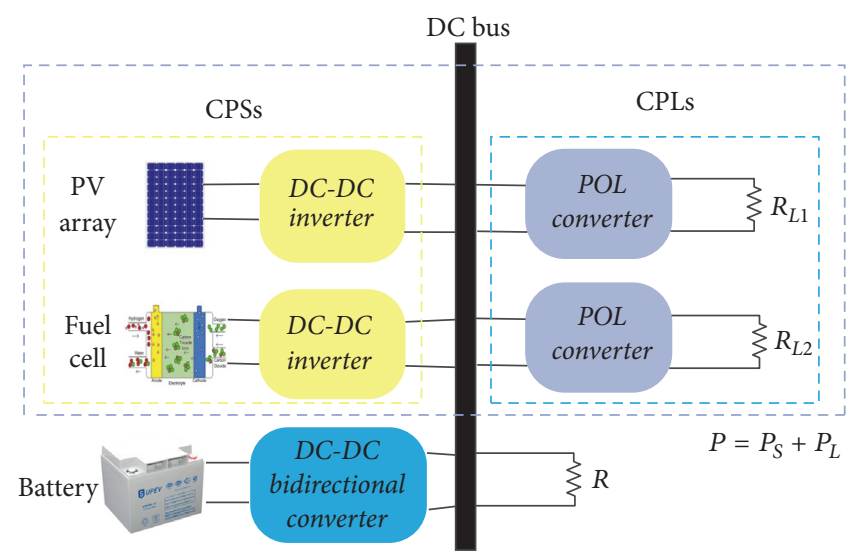

FIGURE 1: Structure of isolated DC microgrid.

such as MGs, telecommunications, plug-in electric vehicle, aerospace, and so on.

The paper is organized as follows. In Section 2, the configuration and large-signal model of the DC MG under isolated operation mode is described. Section 3 presents the theoretical derivations and design of a fixed-frequency PWM-based ASMC for stand-alone DC MG. Also, a detailed analysis of the stability problem is provided in this section. Section 4 demonstrates the performances and comparison of two closed-loop control systems adopted different control methods which are HB-based SMC and the proposed control scheme. Finally, Section 5 concludes the article.

\section{DC Microgrid System Description}

2.1. Microgrid Configuration. The structure of an isolated DC MG is depicted in Figure 1, where all DGs, active loads (CPLs), resistive load, and energy storage device are connected to the DC bus. In such power electronic converter dominated systems, tightly regulated point-of-load converters (POLs) paired with motors, actuators, and even simple resistive loads behave as CPLs. The total power of CPLs in MG is $P_{L}$. This means that the power absorbed from sources by CPLs remains constant during system operation. Moreover, DGs (photovoltaic power and fuel cell) and connected DCDC boost converters are together seen as constant-power sources (CPSs). The total power of CPSs is $P_{S}$. This means that, despite the variation on the bus voltage, the current provided by the power converter adapts to keep injecting a constant power into MG. As CPLs and CPSs behave in the same way, they can be modelled as a lumped CPL, calculated as $P=P_{L}+P_{S}$ [27].

Figure 2 shows the energy flow of DC MG. It can be seen from Figure 2 that the directions of power flow through CPSs, CPLs, and resistive load are unidirectional. The directions of power flow through CPSs are from power sources to the DC bus; inversely, the directions of CPLs and resistive load are from DC bus to loads. However, the power flow direction in bidirectional buck/boost converter is bidirectional. When the direction of the power flow is from battery to the DC bus, it is obvious that the power provided by sources is insufficient supply to meet the load demand. Therefore, the battery is in 


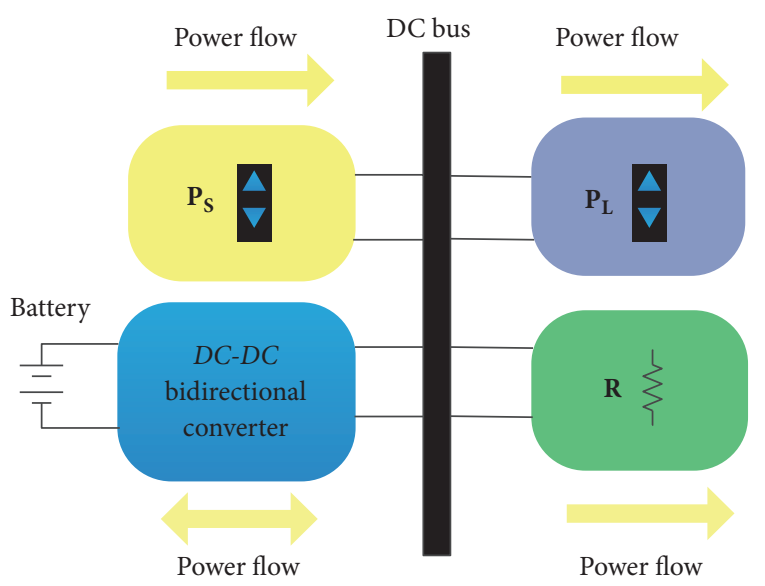

Figure 2: Power flow of DC microgrid.

discharge state, $P \geq 0$. On the other hand, when the power offered by sources is more than load demands, the battery is in charge state, $P \leq 0$. At this time the direction of power flow is from DC bus to battery and the power sources can supply electric power for entire MG system.

2.2. Microgrid Modelling. With purpose of theoretical analysis, an equivalent topology of DC MG is applied in Figure 3. The bus current is described as follows:

$$
\begin{aligned}
i_{\text {bus }} & =i_{R}+i_{P}, \\
i_{R} & =\frac{v_{\text {bus }}}{R}, \\
i_{P} & =\frac{P}{v_{\text {bus }}},
\end{aligned}
$$

where $R$ and $P$ denote the resistance value of resistive load and the power of aforementioned lumped CPL; $i_{R}$ and $i_{P}$ are the currents flowing through resistive load and lumped CPL, respectively. Both $R$ and $P$ are time-variable [28].

Only the case of current continuous conduction mode (CCM) is considered in this paper; hence, during this mode, the dynamic model of MG system is given by

$$
\begin{aligned}
L \frac{d i_{L}}{d t} & =v_{S}-u v_{\text {bus }}, \\
C \frac{d v_{\text {bus }}}{d t} & =u i_{L}-i_{\text {bus }},
\end{aligned}
$$

where $i_{L}, v_{\text {bus }}$, and $v_{s}$ represent inductive current, bus voltage, and battery voltage, respectively. $u$ denotes control input of MG system. $S_{1}$ and $S_{2}$ switch on complementarily. When $S_{1}$ is in conducting state, $u$ is equal to 1 ; conversely, $u$ is equal to 0 . Therefore, the switching control law is adopted as

$$
u=\left\{\begin{array}{l}
u^{+}=1, \\
u^{-}=0 .
\end{array}\right.
$$

For the simplified analysis, we replace $i_{L}$ and $v_{\text {bus }}$ by $x_{1}$ and $x_{2}$, then (2) would be shown as

$$
\dot{x}_{1}=\frac{v_{S}}{L}-\frac{u x_{2}}{L},
$$

$$
\dot{x}_{2}=\frac{u x_{1}}{C}-\frac{M x_{2}}{C}-\frac{N}{C x_{2}},
$$

where $M=1 / R \in \mathbb{R}^{+}, N=P \in \mathbb{R}$, and $v_{S} \in \mathbb{R}^{+}$.

\section{Control Scheme}

3.1. Adaptive Observer. The adaptive observer is gaining increasing attention due to its low requirement to the accuracy of system mathematical model. In addition, this observer has an excellent robustness to variable parameters and external disturbances. In view of the above advantages existing in adaptive observer, based on the theory of parameter estimation, this paper designs a following adaptive observer according to the MG model and its feature:

$$
\begin{aligned}
& \dot{\hat{x}}_{1}=\frac{\widehat{v}_{S}}{L}-\frac{u \widehat{x}_{2}}{L}-K_{1}\left(x_{1}-\widehat{x}_{1}\right), \\
& \dot{\hat{x}}_{2}=\frac{u \widehat{x}_{1}}{C}-\frac{\widehat{M} x_{2}}{C}-\frac{\widehat{N}}{C x_{2}}+K_{2}\left(x_{2}-\widehat{x}_{2}\right),
\end{aligned}
$$

where observer gains are $K_{1}>0$ and $K_{2}>0 ; \widehat{x}_{1}, \widehat{x}_{2}, \widehat{M}, \widehat{N}$, and $\widehat{v}_{S}$ denote the estimates of $x_{1}, x_{2}, M, N$, and $v_{S} \cdot x_{1}$ and $x_{2}$ are observable state variables. Let $\widetilde{x}_{1}=x_{1}-\widehat{x}_{1}, \widetilde{x}_{2}=x_{2}-\widehat{x}_{2}$, $\widetilde{M}=M-\widehat{M}, \widetilde{N}=N-\widehat{N}$, and $\widetilde{v}_{S}=v_{S}-\widehat{v}_{S}$, then the error equations of adaptive observer can be written as follows:

$$
\begin{aligned}
& \dot{\tilde{x}}_{1}=\frac{\widetilde{v}_{S}}{L}-\frac{u \tilde{x}_{2}}{L}-K_{1} \tilde{x}_{1}, \\
& \dot{\tilde{x}}_{2}=\frac{u \widetilde{x}_{1}}{C}-\frac{\widetilde{M} x_{2}}{C}-\frac{\widetilde{N}}{C x_{2}}-K_{2} \widetilde{x}_{2} .
\end{aligned}
$$

Select a positive definite Lyapunov function as the following form:

$$
V=\frac{1}{2} L \widetilde{x}_{1}^{2}+\frac{1}{2} C \widetilde{x}_{2}^{2}+\frac{1}{2 \gamma_{1}} \widetilde{M}^{2}+\frac{1}{2 \gamma_{2}} \widetilde{N}^{2}+\frac{1}{2 \gamma_{3}} \widetilde{v}_{S}^{2},
$$

where the adaptive gains satisfy $\gamma_{1}>0, \gamma_{2}>0$ and $\gamma_{3}>0$. The derivative of Lyapunov function is calculated by

$$
\dot{V}=L \widetilde{x}_{1} \dot{\tilde{x}}_{1}+C \widetilde{x}_{2} \dot{\tilde{x}}_{2}+\frac{1}{\gamma_{1}} \widetilde{M} \dot{\bar{M}}+\frac{1}{\gamma_{2}} \widetilde{N} \dot{\bar{N}}+\frac{1}{\gamma_{3}} \widetilde{v}_{S} \dot{\tilde{v}}_{S} .
$$

Using (6), the derivative of the Lyapunov function can be derived as

$$
\begin{aligned}
\dot{V}= & -K_{1} L{\tilde{x}_{1}}^{2}-K_{2} C \widetilde{x}_{2}^{2}-\widetilde{M}\left(x_{2} \tilde{x}_{2}+\frac{\dot{\bar{M}}}{\gamma_{1}}\right) \\
& -\widetilde{N}\left(\frac{\widetilde{x}_{2}}{x_{2}}+\frac{\dot{\hat{N}}}{\gamma_{2}}\right)+\widetilde{v}_{S}\left(\widetilde{x}_{1}-\frac{1}{\gamma_{3}} \dot{\hat{v}}_{S}\right) .
\end{aligned}
$$

To eliminate the influence of uncertainties in the right side of (9) on state convergence, adaptive rates are structured. These adaptive rates are utilized to eliminate the influence 


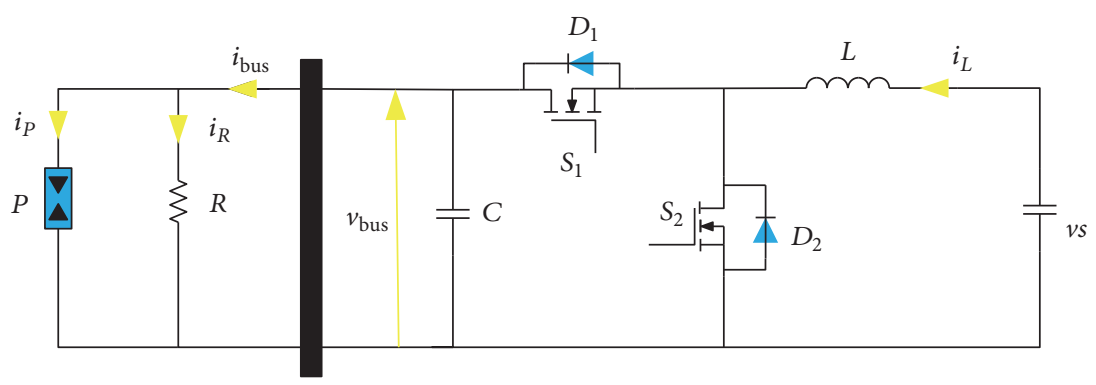

FIGURE 3: Equivalent topology of DC microgrid.

of unknown bounds on the convergence of state error estimation; thus, they are selected as follows:

$$
\begin{aligned}
& \dot{\hat{M}}=-\gamma_{1} x_{2} \tilde{x}_{2}, \\
& \dot{\hat{N}}=-\gamma_{2} \frac{\tilde{x}_{2}}{x_{2}}, \\
& \dot{\hat{v}}_{S}=\gamma_{3} \tilde{x}_{1} .
\end{aligned}
$$

We can get $\dot{V}=-K_{1} L \tilde{x}_{1}{ }^{2}-K_{2} C \widetilde{x}_{2}{ }^{2} \leq 0$, which means $\dot{V}$ is negative semidefinite. Further, based on the Lasalle invariant theorem, $\widetilde{x}_{1}$ and $\widetilde{x}_{2}$ converge to 0 asymptotically.

3.2. ASMC. For the state space $x=\left\{x_{1}, x_{2}\right\} \in \mathbb{R}^{2}$, there exists a discontinuity manifold $\sum:\left\{x \in \mathbb{R}^{2}: S(x)=0\right\}$, which divides state space into two parts by using the time-varying scalar sliding-mode switching function described as

$$
S(x)=S=\widehat{x}_{1}-x_{1 \_ \text {ref }},
$$

where $x_{1 \text { ref }}$ denotes reference value of $x_{1}$.

In this paper, the current estimation error is selected as a sliding-mode switching function. Since the steady-state error of bus voltage is very small, and there is no need to add a voltage error integration term to the sliding surface. This switching function is a time-variant sliding surface, which is different from the traditional fixed-length sliding surface. It would utilize characteristics of adaptive sliding observer to achieve the function of sliding control. On the other hand, since the parameters of switching function are adaptive timevariant, the system would be in the sliding mode at $t=0$ and keep sliding to the expected state on sliding surface with initial conditions selected appropriately. All above facts can realize the quickness and accuracy of control performance.

When system reaches its steady state, based on conservation of energy, the reference value of inductive current can be calculated as follows:

$$
I_{L_{-} \text {ref }}=\frac{V_{\text {bus_ref }}^{2} M+N}{v_{S}},
$$

where $I_{L_{\text {_ref }}}$ and $V_{\text {bus_ref }}$ denote reference values of inductive current and bus voltage at steady state, respectively. And $x_{1 \text { ref }}$ can be obtained as

$$
x_{1 \_ \text {ref }}=V_{\text {bus_ref }}^{2} \frac{\widehat{M}}{\widehat{v}_{S}}+\frac{\widehat{N}}{\widehat{v}_{S}} .
$$

Therefore, the derivative of $S$ can be described by

$$
\begin{aligned}
\dot{S}= & \frac{\widehat{v}_{S}}{L}-\frac{u \widehat{x}_{2}}{L}+K_{1} \widetilde{x}_{1}-\frac{\dot{\hat{N}} \widehat{v}_{S}-\widehat{N} \dot{\hat{v}}_{S}}{\widehat{v}_{S}^{2}} \\
& -\frac{\dot{\bar{M}} \widehat{v}_{S} V_{\text {bus_ref }}^{2}-\widehat{M} \dot{\hat{v}}_{S} V_{\text {bus_ref }}^{2}}{\widehat{v}_{S}^{2}} .
\end{aligned}
$$

By utilizing invariant condition, the equivalent control can be written in the following form:

$$
\begin{aligned}
u_{\text {eq }}= & \frac{\widehat{v}_{S}+K_{1} L \tilde{x}_{1}}{\widehat{x}_{2}}+\frac{L \gamma_{2} \tilde{x}_{2} \widehat{v}_{S}+L \gamma_{3} \tilde{x}_{1} x_{2} \widehat{N}}{\widehat{v}_{S}^{2} x_{2} \widehat{x}_{2}} \\
& +\frac{L V_{\text {bus_ref }}^{2}\left(\gamma_{1} x_{2} \tilde{x}_{2} \widehat{v}_{S}+\gamma_{3} \tilde{x}_{1} \widehat{M}\right)}{\widehat{v}_{S}^{2} \widehat{x}_{2}},
\end{aligned}
$$

where $u_{\text {eq }}$ is smooth function of discrete input function $u$. Based on both concept of equivalent control and the necessary and sufficient condition of sliding mode, there exists a following relationship:

$$
0<u_{\text {eq }}<1
$$

Therefore, we can obtain

$$
\begin{aligned}
0<\widehat{v}_{S}+K_{1} L \tilde{x}_{1}+\frac{L \dot{\hat{N}} \widehat{v}_{S}-L \widehat{N} \dot{\hat{v}_{S}}}{\widehat{v}_{S}^{2}} \\
-\frac{L V_{\text {bus_ref }}^{2}\left(\dot{\hat{M}} \widehat{v}_{S}-\widehat{M} \dot{\hat{v}}_{S}\right)}{\widehat{v}_{S}^{2}}<\widehat{x}_{2} .
\end{aligned}
$$

Moreover, initial conditions should meet (18) to guarantee the occurrence of sidling mode at $t=0$; that is, the system state variables are sliding on sliding surface initially. For control system, there has been $\dot{S}(x)=0$.

$$
\begin{aligned}
\widehat{x}_{2}(0) & >0, \\
\widehat{v}_{S}(0) & >0, \\
\widehat{M}(0) & >0, \\
S(0) & =\widehat{x}_{1}(0)-x_{1 \_ \text {ref }}(0)=0 .
\end{aligned}
$$




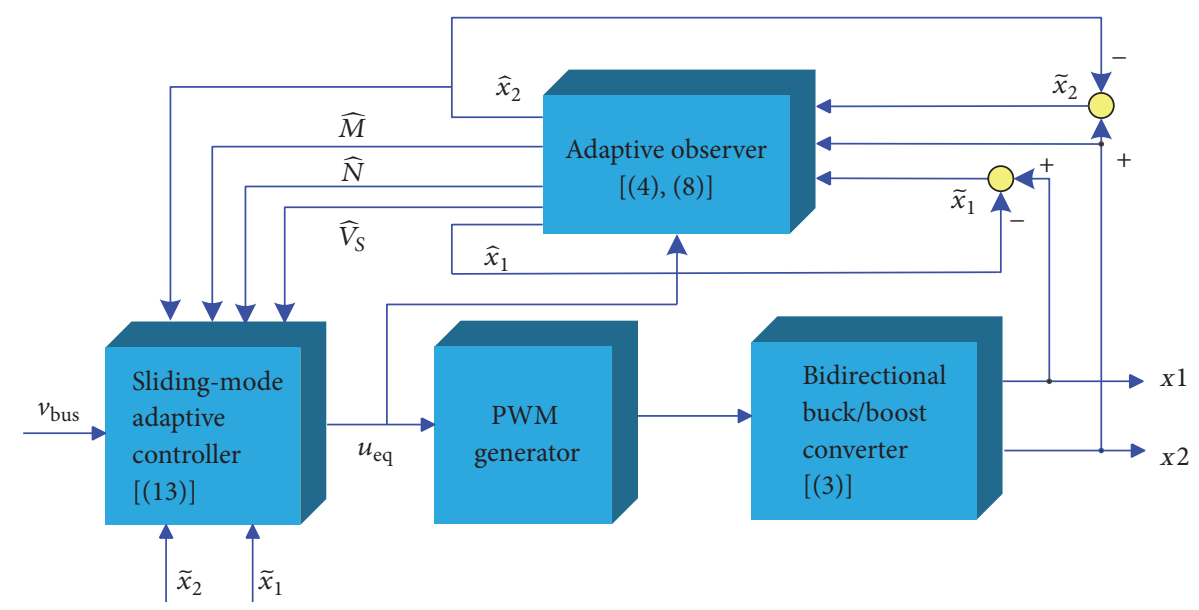

FIGURE 4: Block diagram of control system.

3.3. Composite Control System Structure. The control system structure of microgrid is designed in Figure 4 . The system includes four components: model of microgrid, adaptive observer, sliding adaptive controller, and PWM generator. Each part has the corresponding expression.

SMC is applied into this paper, since the feature of itself variable structure agrees with the characteristic of converter. However, two important characteristics existing in SMC are the switching frequency variation and chattering phenomenon. The chattering problem can be effectively improved by using the ASMC. There are two methods to overcome the defects of variable switching frequency as well as take advantage of SMC simultaneously. One is to add additional hardware circuit to fix switching frequency. It is obviously that this method will increase the cost of system and is adverse to the scalability and plug-play of the distributed generators and loads in DC MG. The other scheme is to combine SMC with other control methods. Since the model used in this paper is large-signal model, the common PWM voltage/current mode control is unsuitable to large-signal situation. Moreover, PWM controller can not show an absolute robustness to input and output variation. Consequently, fixed-frequency PWM-based ASMC, a combination of SMC, PWM method, and adaptive control method, is adopted in this paper. Not only is the influence of power supplies and loads fluctuation on the stability of the system considered, but also the problem that the controller cannot be realized when the input and output are variable and unknown is conquered. In addition, this controller achieves its optimal performance at the possible conditions $[29,30]$.

Fixed-frequency PWM-based ASMC is equivalent to a voltage and current dual-loop control using PWM modulation. Particularly, it is unnecessary for this control method to establish small-signal model and to design the control parameters by utilizing the magnitude-frequency characteristic in classical control theory, so that the design process is relatively simple. Moreover, the proposed control method is derived from modelling of system large signal, which is suitable to wide range of the signal disturbance. Fixed-frequency PWMbased ASMC method has similar fundamental principles with classical PWM methods; the frequency of switching signals is fixed by fastening the frequency of triangle-wave signal. In order to apply the proposed control strategy in DC MG system, it is necessary to convert smoothing function $\left(u_{\text {eq }}\right)$ to the instantaneous duty cycle of the converter represented by $\mathrm{D}$; then $\mathrm{D}$ is used in the bidirectional buck/boost converter to realize the proposed control scheme [31].

\section{Stability Analysis}

Proposition 1. In sliding mode, the closed-loop MG control system consisting of (4)-(6), (10), and (15) is asymptotically stable, and state variables $\left(x_{1}, x_{2}\right)$ converge to the desired equilibrium $\left(I_{L_{-} \text {ref }}, V_{\text {bus_ref }}\right)$.

Proof. By substituting $x_{1}=\tilde{x}_{1}+\hat{x}_{1}, x_{2}=\tilde{x}_{2}+\hat{x}_{2}$, (10), (15), and $\dot{S}(x)=0$ into (5), we obtain

$$
\begin{aligned}
\dot{\hat{x}}_{2} & =\frac{1}{C \widehat{x}_{2}}\left(V_{\text {bus_ref }}^{2} \widehat{M}+V_{\text {bus_ref }}^{2} K_{1} L \tilde{x}_{1} \frac{\widehat{M}}{\widehat{v}_{S}}\right. \\
& +K_{1} L \tilde{x}_{1} \frac{\widehat{N}}{\widehat{v}_{S}}+\frac{L V_{\text {bus_ref }}^{2} \gamma_{3} \widetilde{x}_{1} \widehat{M} \widehat{N}}{\widehat{v}_{S}^{3}} \\
& +\frac{L V_{\text {bus_ref }}^{2} \gamma_{2} \tilde{x}_{2} \widehat{M}}{\widehat{v}_{S}^{2} x_{2}}+\frac{L \gamma_{3} \widetilde{x}_{1} \widehat{N}^{2}}{\widehat{v}_{S}^{3}} \\
& +\frac{L V_{\text {bus_ref }}^{2} \widehat{N} \gamma_{3} \widetilde{x}_{1} \widehat{M}}{\widehat{v}_{S}^{3}}+\frac{L V_{\text {bus_ref }}^{2} \gamma_{1} x_{2} \widetilde{x}_{2} \widehat{N}}{\widehat{v}_{S}^{2}} \\
& +\frac{L \gamma_{2} \widehat{N} \widetilde{x}_{2}}{\widehat{v}_{S}^{2} x_{2}}+\frac{L V_{\text {bus_ref }} \gamma_{1} x_{2} \widetilde{x}_{2} \widehat{M}}{\widehat{v}_{S}^{2}} \\
& \left.+\frac{L V_{\text {bus_ref }}^{4} \gamma_{3} \widetilde{x}_{1} \widehat{M}^{2}}{\widehat{v}_{S}^{3}}+\widehat{N}\right)-\frac{\widehat{M}\left(\widetilde{x}_{2}+\widehat{x}_{2}\right)}{C} \\
& -\frac{\widehat{N}}{C\left(\widetilde{x}_{2}+\widehat{x}_{2}\right)}+K_{2} \tilde{x}_{2} .
\end{aligned}
$$


TABLE 1: Simulation parameters.

\begin{tabular}{lcc}
\hline Name & Parameter & Value \\
\hline Bus reference voltage & $V_{\text {bus_ref }}$ & $380 \mathrm{~V}$ \\
Battery nominal voltage & $v_{S}$ & $300 \mathrm{~V}$ \\
Capacitance & $C$ & $1000 \mu \mathrm{F}$ \\
Inductance & $L$ & $5 \mathrm{mH}$ \\
Switching frequency & $f_{S}$ & $20 \mathrm{kHz}$ \\
Resistive loads & $R$ & $100 \Omega$ \\
\hline
\end{tabular}

Therefore, we yield the following equation:

$$
\begin{aligned}
& \widehat{x}_{2} \dot{\bar{x}}_{2}=\frac{\widehat{M}}{C}\left(V_{\text {bus_ref }}^{2}-\widehat{x}_{2}^{2}\right)+\frac{1}{C}\left(V_{\text {bus_ref }}^{2} K_{1} L \frac{\widehat{M}}{\widehat{v}_{S}}\right. \\
& +K_{1} L \frac{\widehat{N}}{\widehat{v}_{S}}+\frac{L V_{\text {bus_ref }}^{4} \gamma_{3} \widehat{M}^{2}}{\widehat{v}_{S}^{3}}+\frac{L V_{\text {bus_ref }}^{2} \widehat{N} \gamma_{3} \widehat{M}}{\widehat{v}_{S}^{3}} \\
& \left.+\frac{L V_{\text {bus_ref }}^{2} \gamma_{3} \widehat{M} \widehat{N}}{\widehat{v}_{S}^{3}}+\frac{L \gamma_{3} \widehat{N}^{2}}{\widehat{v}_{S}^{3}}\right) \widetilde{x}_{1} \\
& +\frac{1}{C}\left(\frac{L V_{\text {bus_ref }}^{4} \gamma_{1} x_{2} \widehat{M}}{\widehat{v}_{S}^{2}}+\frac{L \gamma_{2} \widehat{N}}{\widehat{v}_{S}^{2}}+\frac{L V_{\text {bus_ref }}^{2} \gamma_{2} \widehat{M}}{x_{2} \widehat{v}_{S}^{2}}\right. \\
& \left.+\frac{L V_{\text {bus_ref }}^{2} \gamma_{1} x_{2} \widehat{N}}{\widehat{v}_{S}^{2}}-\widehat{M} \widehat{x}_{2}+K_{2} C \widehat{x}_{2}\right) \widetilde{x}_{2}+\frac{1}{C}(\widehat{N} \\
& \left.+\frac{\widehat{N} \widehat{x}_{2}}{\widetilde{x}_{2}+\widehat{x}_{2}}\right) \cdot
\end{aligned}
$$

After rearranging terms, (20) is rewritten as

$$
\frac{d \widehat{x}_{2}^{2}}{d t}=\frac{2 \widehat{M}}{C}\left(V_{\text {bus_ref }}^{2}-\widehat{x}_{2}^{2}\right)+\psi+h,
$$

where $h$ and $\psi$ are determined by

$$
\begin{aligned}
h= & \frac{2}{C}\left(\widehat{N}-\frac{\widehat{x}_{2} \widehat{N}}{\widetilde{x}_{2}+\widehat{x}_{2}}\right), \\
\psi= & \frac{2}{C \widehat{V}_{S}}\left(V_{\text {bus_ref }}^{2} K_{1} L \widehat{M}+\frac{L V_{\text {bus_ref }}^{4} \gamma_{3} \widehat{M}^{2}}{\widehat{v}_{S}^{2}}\right. \\
& +\frac{L V_{\text {bus_ref }}^{2} \gamma_{3} \widehat{M} \widehat{N}}{\widehat{v}_{S}^{2}}+K_{1} L \widehat{N}+\frac{L V_{\text {bus_ref }}^{2} \widehat{N} \gamma_{3} \widehat{M}}{\widehat{v}_{S}^{2}} \\
& \left.+\frac{L \gamma_{3} \widehat{N}^{2}}{\widehat{v}_{S}^{2}}\right) \widetilde{x}_{1}+\frac{2}{C \widehat{v}_{S}^{2}}\left(L \gamma_{2} \widehat{N}+L V_{\text {bus_ref }}^{4} \gamma_{1} x_{2} \widehat{M}\right. \\
& +L V_{\text {bus_ref }}^{2} \gamma_{2} \frac{\widehat{M}_{x_{2}}}{x_{2}} \widehat{x}_{2} \widehat{v}_{S}^{2} \widehat{M}+K_{2} C \widehat{x}_{2} \widehat{v}_{S}^{2} \\
& \left.+L V_{\text {bus_ref }}^{2} \gamma_{1} x_{2} \widehat{N}_{S}\right) \widetilde{x}_{2} .
\end{aligned}
$$

Since $\tilde{x}_{1}$ and $\tilde{x}_{2}$ converge to $0, \psi$ and $h$ converge to 0 ; thus, $\widehat{x}_{2}$ converges to $\pm V_{\text {bus_ref }}$. In addition, according to (17),

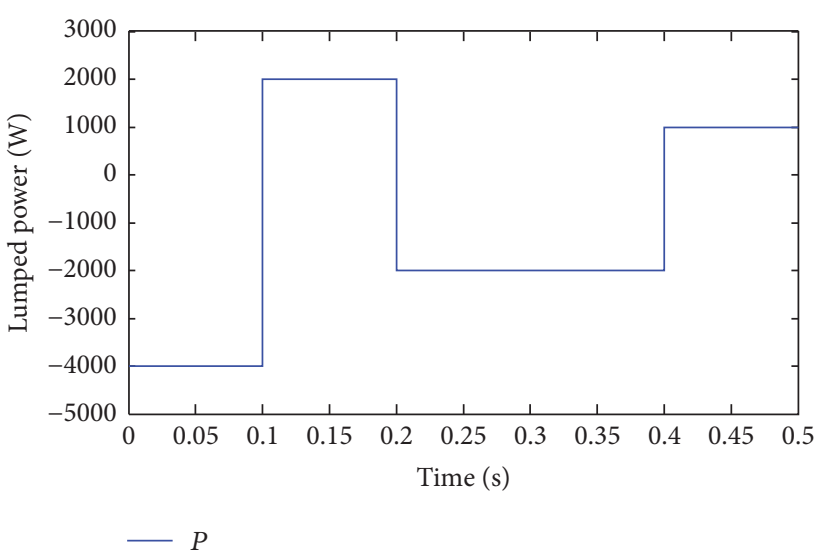

FIGURE 5: Change of lumped power.

$\hat{x}_{2}>0$; therefore, it is obvious that $\hat{x}_{2}$ and $x_{2}$ converge asymptotically to $V_{\text {bus_ref }}$. By substituting (15) into (4), we can obtain $x_{L_{-} \text {ref }}$ converges to $I_{L_{-} \text {ref }}$; thus $\widehat{x}_{1}$ and $x_{1}$ converge to $I_{L \_ \text {ref }}$.

Remark 2. In order to realize the fixed-frequency PWMbased SMC in bidirectional buck/boost converter, it is necessary to transform the smooth function to the demand instantaneous duty ratio of converter [32].

\section{Simulation}

To validate the feasibility of the proposed adaptive slidingmode controller in islanded DC MG, a real-time simulation circuit is built in MATLAB. The simulation parameters are shown in Table 1.

The observer gains are $K_{1}=K_{2}=685$ with the adaptive gains $\gamma_{1}=3, \gamma_{2}=150$, and $\gamma_{3}=150$. The initial conditions are selected as $\widehat{x}_{2}(0)=365 \mathrm{~V}, \widehat{v}_{S}(0)=303 \mathrm{~V}, \widehat{M}(0)=1 \times 10^{-2}$, $\widehat{N}(0)=-4000 \mathrm{~W}$, and $\widehat{x}_{1}(0)=-8.44 \mathrm{~A}$. The sliding mode occurs at $t=0$, so that $S(0)=0$.

DGs and CPLs are the primary factors that affect the stability of MG, so simulation has been conducted under the following operating conditions. Four-step changes were applied in the power $P$, shown in Figure 5.

$$
\begin{aligned}
& \text { 1. } t=0 \mathrm{~s}, P=-4000 \mathrm{~W} ; \\
& \text { 2. } t=0.1 \mathrm{~s}, P=-4000 \mathrm{~W} \rightarrow 2000 \mathrm{~W} ; \\
& \text { 3. } t=0.2 \mathrm{~s}, P=2000 \mathrm{~W} \rightarrow-2000 \mathrm{~W} ; \\
& \text { 4. } t=0.4 \mathrm{~s}, P=-2000 \mathrm{~W} \rightarrow 1000 \mathrm{~W}
\end{aligned}
$$




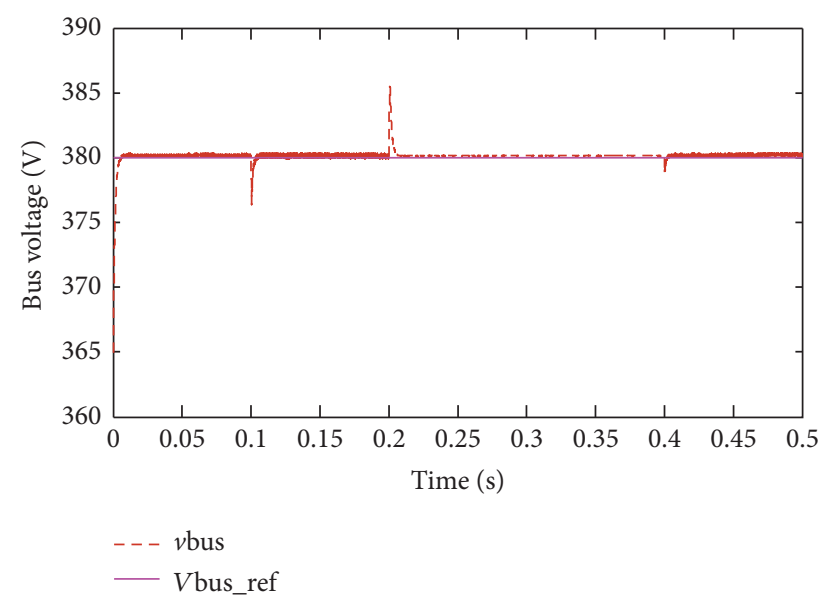

FIGURE 6: DC bus voltage under hysteresis band-based SMC.

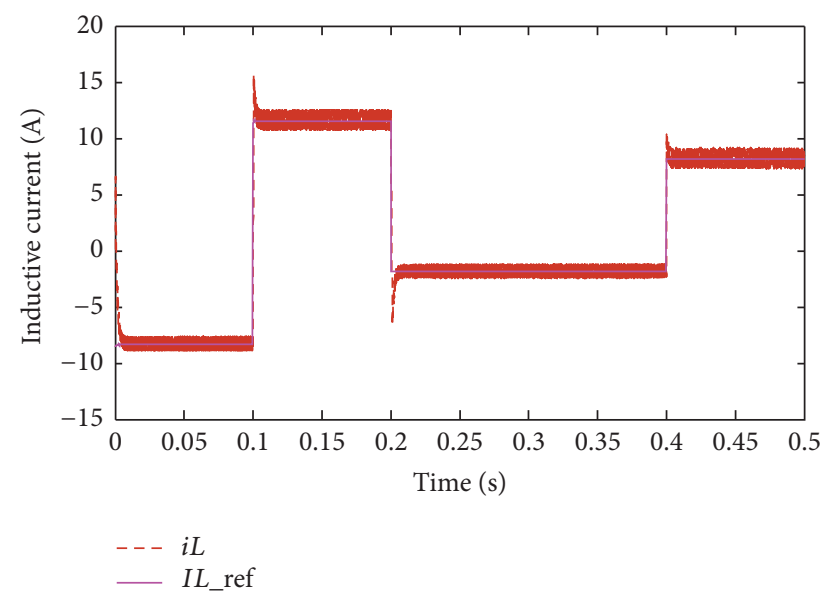

FIGURE 7: Inductive current under hysteresis band-based SMC.

5.1. Hysteresis Band-Based SMC. In this scenario, SMC is adopted in DC MG system and $\mathrm{HB}$ method is applied to limit the switching frequency. The real-time simulation response of the system corresponding to this control method is shown in Figures 6-8. It can be seen from Figure 6, under the impact of CPLs, which the bus voltage reaches to its reference value in $20 \mathrm{~ms}$ with larger transient values and chattering amplitude, voltage fluctuations in the range of $-1.2 \% \sim+1.6 \%$. The inductive current tracks its reference value perfectly but has high startup value, chattering amplitude, and step values at the turning points, showed in Figure 7. The controller ensures DC bus voltage regulation within permissible limits in the case of the power $P$ mutation. However, the adopted frequency limit method can solve high switching frequency problem; it can do nothing to make the frequency constant. Thus, this approach makes it difficult to design the input and output filter. Figure 8 shows the charge and discharge status of battery. When the power flows from battery to the DC bus $(P \geq 0)$, the battery is in discharge state. On the contrary, when the power flows from DC bus to battery $(P \leq 0)$, the battery is in charge state.

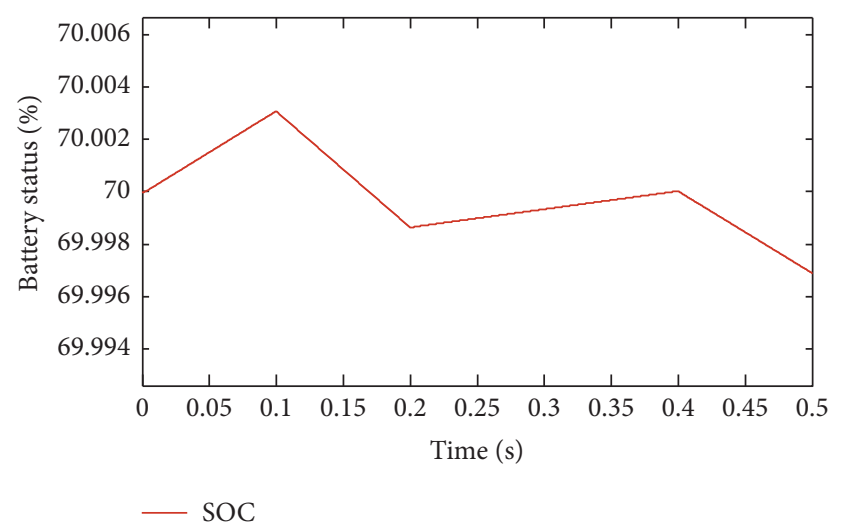

FIGURE 8: Battery status under hysteresis band-based SMC.

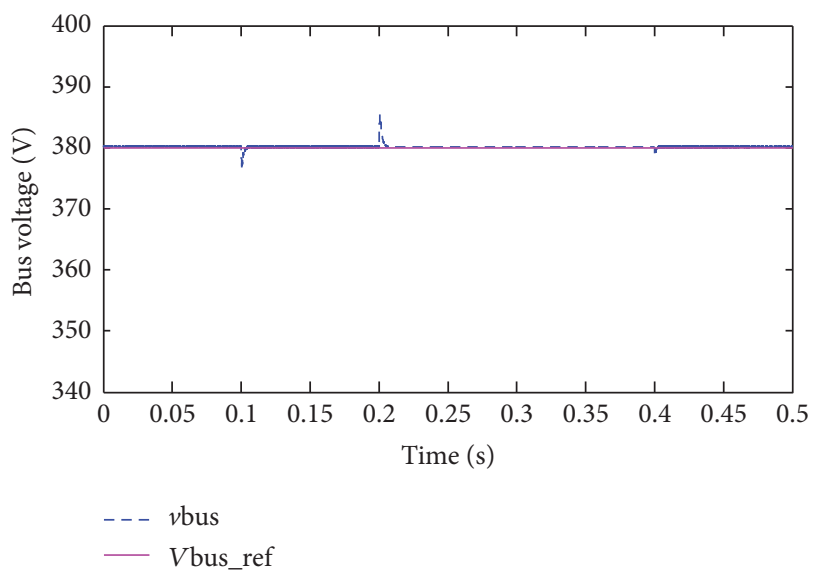

FIGURE 9: DC bus voltage under fix-frequency PWM-based ASMC.

5.2. Fix-Frequency PWM-Based ASMC. In this scenario, response waveforms of MG system are shown in Figures 9-11, which describe DC bus voltage, inductive current, and state of charge, respectively. It can be seen from Figure 9, under the $380 \mathrm{~V}$ bus reference voltage, which voltage state variable can trace the reference voltage within $8 \mathrm{~ms}$. Moreover, fluctuation range of bus voltage is $-1 \% \sim 1.3 \%$ in the case of CPLs mutation. That is, the adaptive sliding-mode controller ensures both the bus voltage fluctuating at a permissible range and converging to reference value at a fast rate. Compared with the results in Figure 6, the maximum fluctuation range of $\mathrm{dc}$ bus voltage has been reduced $0.3 \%$ with less than $5 \%$ allowable range of voltage fluctuation. The voltage regulation time has also been shortened $12 \mathrm{~ms}$. In addition, it can be seen from the smooth output waveform that the chattering phenomenon has been effectively improved. Figure 10 shows that inductive current can trace the reference current perfectly starting from the initial state. Although there is a slight change in the inductor current in the case of CPLs mutation, it is quickly adjusted to the reference value. Compared with the results in Figure 7, the maximum fluctuation range and regulation time of inductive current have been substantially reduced. It can be seen from the smooth output current waveform that the chattering phenomenon has been effectively improved. Accordingly, the system state variables have 


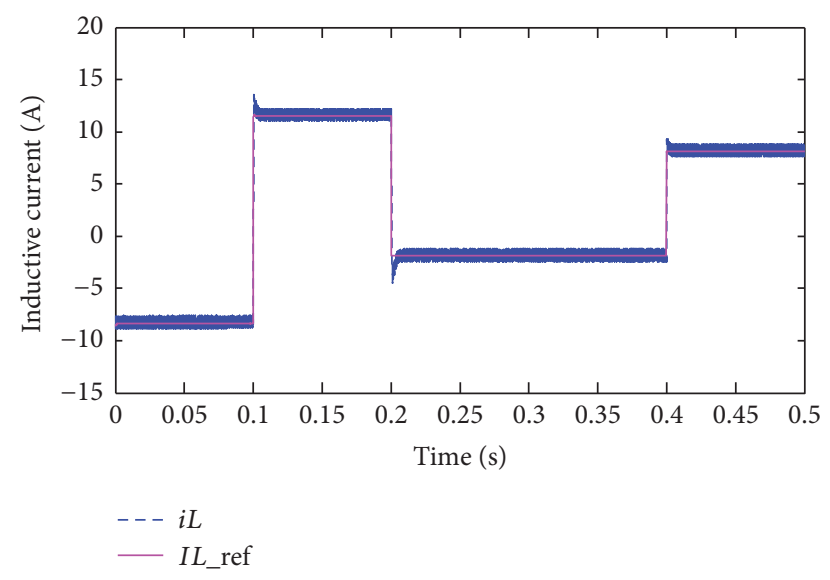

FIGURE 10: Inductive current under fix-frequency PWM-based ASMC.

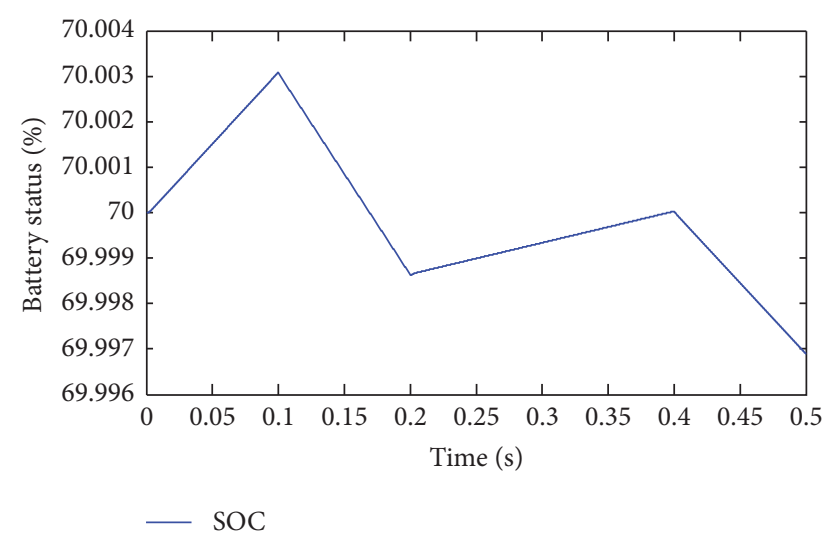

FIGURE 11: Battery status under fix-frequency PWM-based ASMC.

been well maintained on the sliding surface and the dynamic response and transient values of inductive current have been improved in the whole process. Figure 11 shows the charge and discharge status of battery. The overall trend is similar to that in Figure 8. The change of battery status agrees with the trend of $P$ variation. When $P$ is less than 0 , the battery is in charge status; otherwise, battery is in discharge status. In addition, the PWM is used in this control method to make the frequency constant; thus, the switching frequency of the system is the same as that of the triangular wave.

Overall, it can be seen that the designed control system has a good robustness to deal with the parameters uncertainty and CPLs of DC system, and there is a good match among the theoretical analysis and simulation.

\section{Conclusions}

An ASMC-based bus voltage control strategy was proposed in this paper, which is especially suitable for an isolated DC MG. With the proposed control strategy, bus voltage can be fast and accurately tracked without additional sensors/hardware circuits and high bandwidth communications, which is key for the system scalability and maintaining the plug-andplay feature of the DGs. By using the ASMC method to estimate the uncertainty and external disturbance (CPL et al.), the system parameters and switching function can be adjusted in time to weaken the chattering. Based on the ASMC, the fix-frequency PWM-based ASMC can make the frequency constant, which greatly reduces the difficulty of the design of the filter. The simulation results show that the designed controller has the robustness to large disturbance. Moreover, the proposed MG control method is of significance to simplify the design of MG control system, to reduce the costs of MG projects and to provide stable power supply for island, remote areas without electricity, and other demand areas.

\section{Conflicts of Interest}

The authors declare that they have no conflicts of interest.

\section{Acknowledgments}

This work was supported in part by the National Nature Science Foundation of China (Grant no. 61374155). The research is also supported by Research Fund for the Doctoral Program of Higher Education of China (Grant no. 20130073110030).

\section{References}

[1] B. Thomsen, J. M. Guerrero, and P. B. Thøgersen, "Faroe islands wind-powered space heating microgrid using self-excited 220$\mathrm{kW}$ induction generator," IEEE Transactions on Sustainable Energy, vol. 5, no. 4, pp. 1361-1366, 2014.

[2] H. Laaksonen, D. Ishchenko, and A. Oudalov, "Adaptive protection and microgrid control design for Hailuoto Island," IEEE Transactions on Smart Grid, vol. 5, no. 3, pp. 1486-1493, 2014.

[3] C. Yuan, M. A. Haj-Ahmed, and M. S. Illindala, "Protection strategies for medium-voltage direct-current microgrid at a remote area mine site," IEEE Transactions on Industry Applications, vol. 51, no. 4, pp. 2846-2853, 2015.

[4] J. Tian, Z. Liu, J. Shu, J. Liu, and J. Tang, "Base on the ultrashort term power prediction and feed-forward control of energy management for microgrid system applied in industrial park," IET Generation, Transmission \& Distribution, vol. 10, no. 9, pp. 2259-2266, 2016.

[5] M. Hong, X. Yu, N.-P. Yu, and K. A. Loparo, "An energy scheduling algorithm supporting power quality management in commercial building microgrids," IEEE Transactions on Smart Grid, vol. 7, no. 2, pp. 1044-1056, 2016.

[6] D. Zhang and J. Wang, "Research on construction and development trend of micro-grid in china," Power System Technol, vol. 40, no. 2, pp. 451-458, 2016.

[7] T. Sakagami, A. Werth, M. Tokoro, Y. Asai, D. Kawamoto, and H. Kitano, "Performance of a DC-based microgrid system in Okinawa," in Proceedings of the 4th International Conference on Renewable Energy Research and Applications (ICRERA '15), pp. 311-316, Paris, France, November 2015.

[8] E. E. Ufluoğlu and G. Kayakutlu, "Mathematical model for a microgrid consisting of wind turbine, PV panels, and energy storage unit," Journal of Renewable and Sustainable Energy, vol. 8, no. 5, Article ID 054101, 2016.

[9] H. Zhang, D. Zhao, C. Gu, and F. Li, "Bilevel economic operation of distribution networks with microgrid integration," 
Journal of Renewable and Sustainable Energy, vol. 7, no. 2, Article ID 023120, 2015.

[10] M. S. Mahmoud, M. S. U. Rahman, and F. M. A. L. Sunni, "Review of microgrid architectures - a system of systems perspective," IET Renewable Power Generation, vol. 9, no. 8, pp. 1064-1078, 2015.

[11] S. Augustine, M. K. Mishra, and N. Lakshminarasamma, "Adaptive droop control strategy for load sharing and circulating current minimization in low-voltage standalone DC microgrid," IEEE Transactions on Sustainable Energy, vol. 6, no. 1, pp. 132141, 2015.

[12] A. Emadi, A. Khaligh, C. H. Rivetta, and G. A. Williamson, "Constant power loads and negative impedance instability in automotive systems: definition, modeling, stability, and control of power electronic converters and motor drives," IEEE Transactions on Vehicular Technology, vol. 55, no. 4, pp. 1112-1125, 2006.

[13] P. Magne, B. Nahid-Mobarakeh, and S. Pierfederici, "Dynamic consideration of DC microgrids with constant power loads and active damping system-a design method for fault-tolerant stabilizing system," IEEE Journal of Emerging and Selected Topics in Power Electronics, vol. 2, no. 3, pp. 562-570, 2014.

[14] M. Hamzeh, H. Karimi, and H. Mokhtari, "A new control strategy for a multi-bus MV microgrid under unbalanced conditions," IEEE Transactions on Power Systems, vol. 27, no. 4, pp. 2225-2232, 2012.

[15] M. Babazadeh and H. Karimi, "A robust two-degree-of-freedom control strategy for an islanded microgrid," IEEE Transactions on Power Delivery, vol. 28, no. 3, pp. 1339-1347, 2013.

[16] R. Ahmadi and M. Ferdowsi, "Improving the performance of a line regulating converter in a converter-dominated DC microgrid system," IEEE Transactions on Smart Grid, vol. 5, no. 5, pp. 2553-2563, 2014.

[17] K. Yu, Q. Ai, S. Wang, J. Ni, and T. Lv, "Analysis and optimization of droop controller for microgrid system based on small-signal dynamic model," IEEE Transactions on Smart Grid, vol. 7, no. 2, pp. 695-705, 2016.

[18] S. Singh, D. Fulwani, and V. Kumar, "Robust sliding-mode control of dc/dc boost converter feeding a constant power load," IET Power Electronics, vol. 8, no. 7, pp. 1230-1237, 2015.

[19] Z. Yang, X. Zha, J. Sun, and F. Liu, "Global stabilization based on feedback linearization for DC microgrid," Electric Power Automation Equipment, vol. 35, no. 10, pp. 10-14, 2015.

[20] M. Cucuzzella, G. P. Incremona, and A. Ferrara, "Design of robust higher order sliding mode control for microgrids," IEEE Journal on Emerging and Selected Topics in Circuits and Systems, vol. 5, no. 3, pp. 393-401, 2015.

[21] S. K. Gudey and R. Gupta, "Recursive fast terminal sliding mode control in voltage source inverter for a low-voltage microgrid system," IET Generation, Transmission \& Distribution, vol. 10, no. 7, pp. 1536-1543, 2016.

[22] A. P. N. Tahim, D. J. Pagano, and E. Ponce, "Nonlinear control of $\mathrm{dc}$-dc bidirectional converters in stand-alone dc Microgrids," in Proceedings of the 51st IEEE Conference on Decision and Control (CDC '12), pp. 3068-3073, 2012.

[23] L. Benadero, R. Cristiano, D. J. Pagano, and E. Ponce, "Nonlinear analysis of interconnected power converters: a case study," IEEE Journal on Emerging and Selected Topics in Circuits and Systems, vol. 5, no. 3, pp. 326-335, 2015.

[24] X. Su, M. Han, and H. Sun, "Stability control strategy for microgrid based on adaptive total sliding-mode Control," CSEE, vol. 34, no. 31, pp. 6720-6729, 2014.
[25] M. Pahlevani, S. Pan, S. Eren, A. Bakhshai, and P. Jain, "An adaptive nonlinear current observer for boost PFC AC/DC converters," IEEE Transactions on Industrial Electronics, vol. 61, no. 12, pp. 6720-6729, 2014.

[26] S. C. Tan, Y. M. Lai, C. K. Tse, and M. K. H. Cheung, "A fixedfrequency pulsewidth modulation based quasi-sliding-mode controller for buck converters," IEEE Trans. Power Electron, vol. 20, no. 6, pp. 1379-1392, 2005.

[27] T. A. P. Nobrega, D. J. Pagano, E. Lenz, and V. Stramosk, "Modeling and stability analysis of islanded DC microgrids under droop control," IEEE Trans. Power Electron, vol. 30, no. 8, pp. 4597-4607, 2015.

[28] N. L. Diaz, T. Dragicevic, J. C. Vasquez, and J. M. Guerrero, "Intelligent distributed generation and storage units for DC microgrids - A new concept on cooperative control without communications beyond droop control," IEEE Transactions on Smart Grid, vol. 5, no. 5, pp. 2476-2485, 2014.

[29] J. Baek, M. Jin, and S. Han, "A new adaptive sliding-mode control scheme for application to robot manipulators," IEEE Transactions on Industrial Electronics, vol. 63, no. 6, pp. 36283637, 2016.

[30] Y. He and F. L. Luo, "Design and analysis of adaptive slidingmode-like controller for DC-DC converters," IEE Proceedings Electric Power Applications, vol. 153, no. 3, pp. 401-410, 2006.

[31] R. Venkataramanan, A. Sabanovic, and S. Cuk, "Sliding mode control of DC-to-DC converters," in Proceedings of the in IEEE Conference Industrial Electronics, Control and Instrumentations, pp. 251-258, San Francisco, California, USA, 1985.

[32] S. Singh and D. Fulwani, "A PWM based sliding-mode control for negative impedance stabilization in DC Micro-girds," in Proceedings of the 6th IEEE Power India International Conference (PIICON '14), pp. 1-6, Delhi, India, December 2014. 


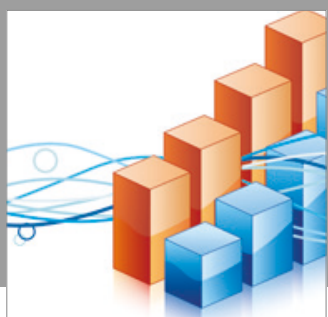

Advances in

Operations Research

vatersals

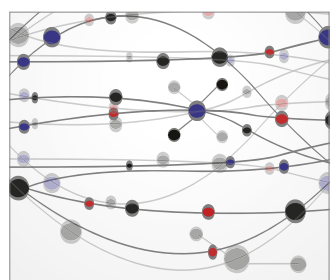

\section{The Scientific} World Journal
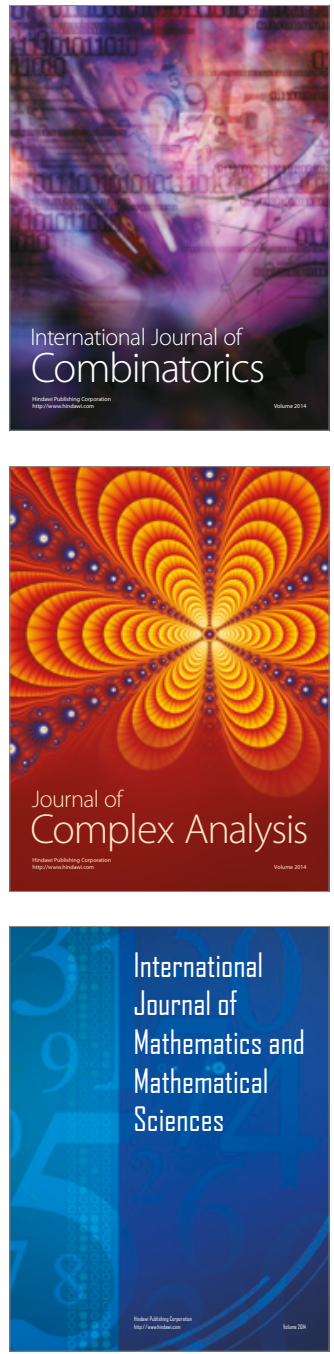
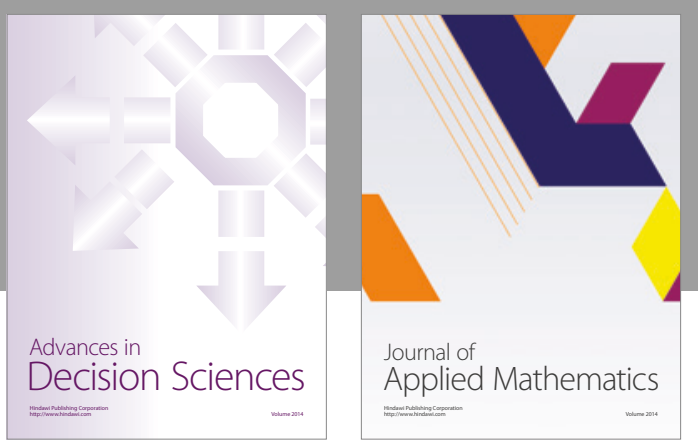

Algebra

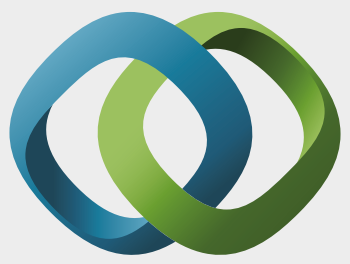

\section{Hindawi}

Submit your manuscripts at

https://www.hindawi.com
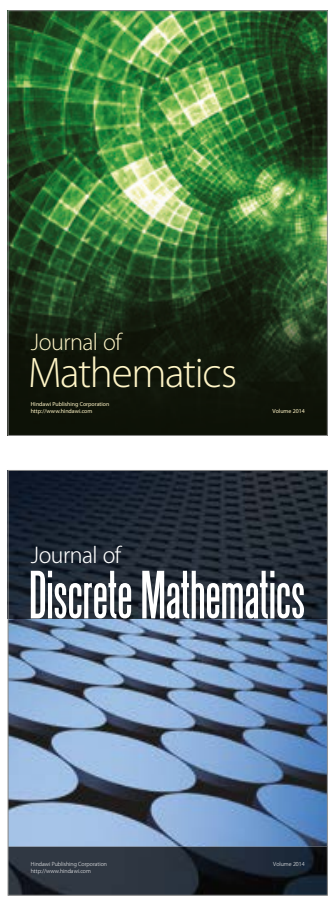

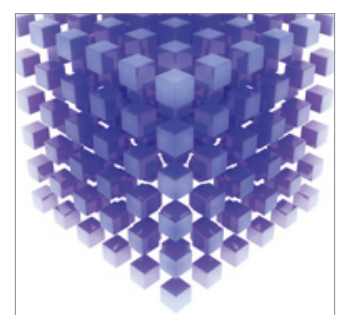

Mathematical Problems in Engineering
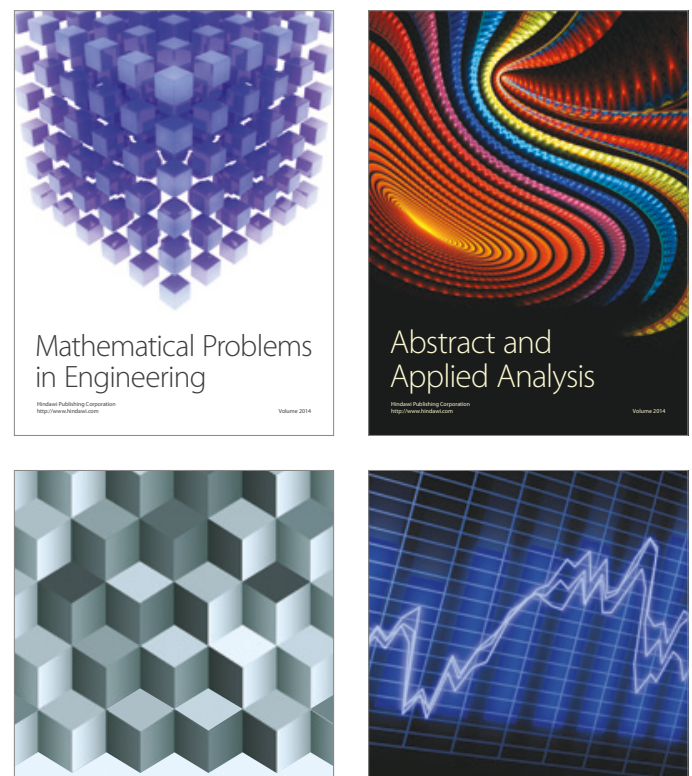

Journal of

Function Spaces

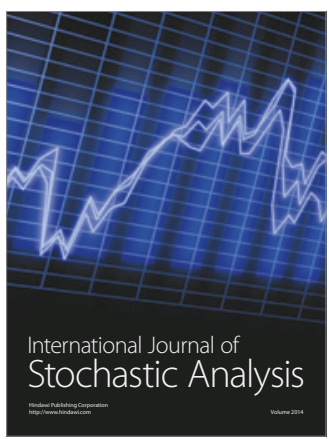

Probability and Statistics
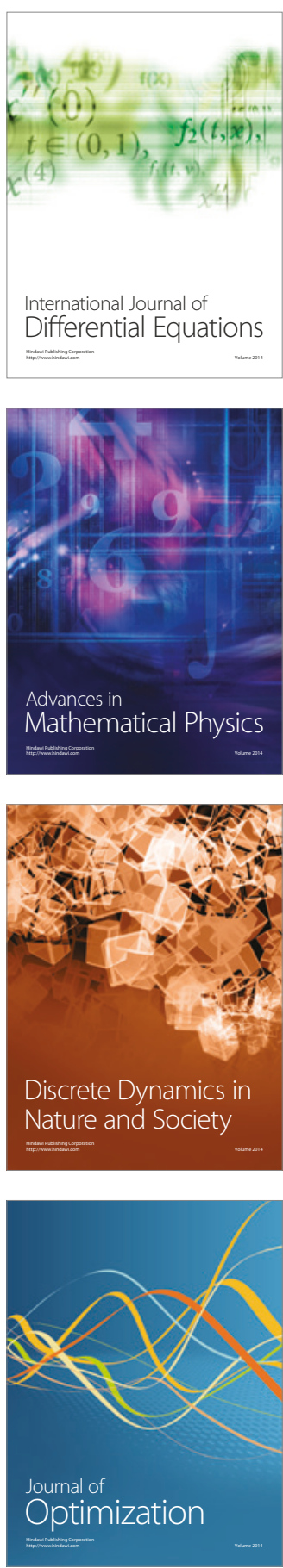